pulsory education of medical students in mental diseases, and the class of psychological medicine is very scantily attended; I might even say it is virtually nonexistent at many of the schools. 2. The qualifying examinations demand no knowledge of mental diseases. 3 . Nine out of ten medical men have never seen a case of iusanity until they are called upon in practice to examine a supposed lunatic and certify if necessary. What, then, I would ask, is the value of such a "skilled" certificate? And yet by virtue of an order signed by a relative or friend and two such certificates the subject can be deprived of his freedom! It being illegal for medical men to act in consultation on the case is certainly also a mistake, and opposed to the golden rule that "two heads are better than one." The origin of this separate examination clause was a desire to prevent collusion. In favour of the adequacy of the present system of certification, it has been stated that the medical officer of an asylum rarely returns a patient thus admitted as "not insane ;" but, I would add, most medical officers are ready to admit that cases not unfrequently occur which have been unnecessarily sent to the asylum. The possible remedies for these defects are as follows :-1. The appointment of district experts as medical officers of insanity occupying analogous posts with that of coroner or medical officer of health, with fixed salaries; these officers to be elected from their experience in the specialty, and to be allowed to practise as pure phtsicians. Their duties would $b$ to examine all supposed lunatics in consultation with the medical man in attendance, to sign all necessary certificates, to visit all single patients and patients in private asylums in their districts, to report thereon from time to time to the Commissioners in Lunacy, and so act as district agents for the Commissioners or Deputy Commissioners. When the fact is taken into consideration that the work of the Lunacy Commission has more than doubled of recent years, its numerical strength remaining unchanged, it will be agreed that this aid will not be unwelcome. The salaries of these officers must be fixed to prevent any pecuniary interest in connexion with the certificates, for at present no fee is forthcoming in many instances unless the certificate be signed. The fees now paid for certification would go to meet the expenses of these new appoin ments. In cases requiring immediate treatment it should be in the power of the medical officer of any asylum to grant an " emergency certificate," under which a patient could be detained for three days, during which time the usual legal course might be pursued. 2. The theoretical and practical study of mental diseases to be made compulsory at the medical schools and by the examining bodies.

iII. Care and Treatment.-A. Pauper patients are congregated in too large numbers in the county and borough asylums for the individual cases to receive the medical attention from day to day that should be allotted to them. It is generally acknowledged that mental diseases are most curable in the early stages, when they require careful treatment. Why then should not patients be received into "hospitals for the insane" of limited size (sav, for from 100 to 200 patients), each having a highly qualified medical staff, with clinical assistants, and all the necessary means of treatment? Cases after a fixed time deemed incurable should be transferred to the "asylums," the "homes for incurables requiring no special medical treatment." Heretofore the lunacy laws have been framed on the score of humanity solely, with the result that the insane have steadily increased in numbers "by accumulation." Surely, it would be more economical, more humane, and more creditable to civilization to endeavour to keep down this accumulation by offering increased facilities for cure. With this end in view, the rati., of medical officers to patients in the hospitals for the insane should be fixed by law, and the large mass of material for practical instruction in mental diseases contained in the county and borough asylums should be placed within the reach of the general body of the medical profession. $B$. Private patients are at the present time largely under the care of medical men who are asylum proprietors. This system, wrong, to say the least of it, from its possibilities of abuse, cannot be defended, and ought to be abolished at the earliest possible date. The asylums of the future for the upper and middle classes will be institutions similar to $\mathrm{St}$ Andrew's Hospital for Mental Diseases, Northampton, and Wonford. House Hospital, near Exeter, managed by Committees, and with efficient medical officers having no direct pecuniary interest in the patients.

IV. Discharge on Recovery or otherwise. $-A$. For pauper patients. The method of discharge by members of the Com. mittee of Visitors, on the recommendation of the medical superintendent, appears in every way satisfactory. $B$. For private paisents. The district medieal officer of insanity should have power to order the discharge of any single patient, or auy patient from any private asylum in his district, should he deem such a course desirable on account of recovery or otherwise. He should also be in a position to prevent the removal of any patient by his or her friends, when such removal is calculated to be fraught with danger to the patient or other persons.

While purposely abstaining from reference to the many good features of the existing lunacy laws, most of which owe their origin to the philanthropy of the legislators of the past, I would fain, in conclusion, remind the legislators of the present that insanity is but the outward expression of bodily defect or disease, and that if the acknowledged accumulation of the insane is to be arrested it can be solely by offering legislative aid to medical science, with the hope that a largely increased percentage of cases of recevery will result therefrom.

Kent Asglum, Chartham.

\section{CASE OF SIMPLE MENINGITIS; NECROPSY; REMARKS.}

By ERNEST F. NEVE, M.B. ED, M.R.C S RESIDENT PHYSICIAN TO THE COWGATE DISPENSARX, EDINBURGH.

I WAs called on Sept. 19th, 1884, to see Mrs. Gaged thirty-six, multipara, seven children, two miscarriages, four of the children dead. Family history good on father's side. Mother died of consumption. A brother who had served in India suffered from sunstroke, had been internperate, and died of "brain fever" after a short illness. The patient had delirium tremens two years ago, but aince then had been temperate. She was six months pregnant.

On Sept. 17th she complained in the middle of the day of a headache. During the night she slept badly, seemed feverish, and complained of her head. On Sept. 18:h she rose early, and worked hard at washing all day. She cane home a little after 7 P.M. complaining of the pain in her head, which was worse. She was quite sensible, but had no appetite, did not sleep that night, complained of shivering, and wandered in her speech once or twice. On Sept. $19 \mathrm{th}$ she was sensible at 6 A.M. About 10 A.M she became delirious, and very soon after sat up and began to grapple with the wall. She continued to struggle, bat did not speak after $11.30 \mathrm{~A} . \mathrm{M}$.

When I saw her for the first time, at 12.30 P.M., she was flinging herself about wildly - one moment throwing herself forward then backwards, then over the edge of the bed, now against the wall, the next moment trying to rise up in bed. Three women of average strength were unable to hold her still. She was breathing very rapidly. The right pupil was found to be contracted, the left of normal size. Large doses of potassium bromide and chloral bydrate were attempted to be given every fifteen minutes. During the administration of this she vomited once, and the bowels were moved. At 2.30 P.M. she was in the same condition, so I placed her under the influence of chloroform. Her temperature was now found to be $101.5^{\circ} \mathrm{F}$; p pulse 120 to 128 per minute, irregular but full ; respiration 60 per minute. At 3 P.M. the pulse was 88 , respiration 60 . Ten ounces of urine were drawn off, high coloured, clear, no albumen. Cervix uteri soft ; ex. ternal os patent, internal closed. The hands were flexed and adducted at the wrists, and the thumbs tucked under the fingers. At 5 P.M. I cut off her hair, applied an icebag to the head, and allowed her to recover from the chloroform. The wild movements recommenced, but not so severely as before. Six minims of morphia (inj. hypod.) were now injected hypoder mically. At 6 P.M. the tem verature was $99.7^{\circ}$ pulse 95 to 100 , full, strong, but irregular; respiration 28 , and heavy. Pupils, left medium, right contracted, and both immovable to light. Lips rather dry; right knee flexed. At $8 \mathrm{P}$.M. the pulse was 96 , respiration 36 . At 10.30 P.M. the temperature was $1002^{\circ}$, pulse 88 , and respiration 28 . Moves all her limbs occasionally; opened her eyes once on a bright light being thrown upon them, and rubbed the left eye upon my attempting an ophthalmoscopic obser. vation. Fairly quiet during the night. On Sept. 20th, at 10 A.M., the temperature was $1012^{\circ}$, pulse 118 to 
10 , a piration 48 ; left pupil more contracted ; movemetids on both sides of the body, more on left than ight, face llushed and conjunctiva somewhat injected. at 1 P.M. the temperature was $1002^{\circ}$, pulse 100 , respiration 50 , coarse râles in throat; moved right arm; legs motionle s and flaccid. She now gradually sank; the pupils trecame almost equal and contracted; by degrees all movements of the limbs subsided ; the breathing became more shallow ; extremities cold ; radial pulse imperceptible; the heart very rapid. At 8 P.M. she expired and death was immediately followed by expulsion of the foetus and placenta, the membranes being intact.

N'ecropsy, thirty-eight hours after death.-Dura mater only slightly adherent to skull cap. On removing the dura mater the vessels of the vertex were seen to be much injected. Beneath the arachnoid and over the entire surface of the brain, especially between the convolutions, there was seropurulent and in some places purulent fluid; pia mater not thickened, but its vessels congested; injecrion especially marked over the junction of the left ascending frontal and parietal convolutious; at the base of the skull there was half an ounce of sero-sanguineous fluid; the venous sinu: es of the base were full of dark semi-fluid blood; the vessels of the base of the brain were healthy; the membranes of the base ap. peared slightly thickened, especially in front of the pons Varolii and along the Sylvian fissures; in the latter there was a great amount of sero-purulent fluid. Careful macroscopic examination failed to reveal the presence of tubercles. On section the brain-substance appeared soft and its vessels were injected; the two lateral ventricles contained half an ounce of blood-stained fluid, with flocculi of softened braintissue.

Remarks. - The etiology of this case is obscure. There may have been some slight tubercular diathesis. The mortality amongst the patient's children was higb, and her mother was consumptive; but Mrs. G- had never manifested this tendency in the slightest degree. She was once intemperate, but not recently so. Intemperance is one of the only well-authenticated causes of simple meningitis. ${ }^{1}$ It is possible that, for obvious reasons, the history was incorrectly stated in this respect. The si mptoms are chitfly striking on account of their severity. The duration of the illness was very short. The violence of the motor excitement exceeds anything which I have seen or read of in connexion with meningitis. The unconsciousness and absolute silence $n f$ the patient in the midst of it all were very remas $k$ able. Vomiting, which is looked upon as a frequent and important symptom, ${ }^{2}$ was practically absent. This fact, which was also noticed by Dr. Lewis Smith in a certain proportion of twenty-one cases of meningitis analysed by him, ${ }^{3}$ is probably due to its being in these cases a phenomenon of special reflex irritability needing some stimulant, such as food or medicine, to set it up. When this is not given, this symptom will of ten be absent. The temperature in meningitis, according to Rosenthal, Wunderlicb, and Rosenstein, maintains high figures. Tanner (loc. cit.) speaks of it as not very high, "seldom reaching $102^{\circ} \mathrm{F}$." This case supports the views of the latter. The tendency to slowing of the previously rapid pulse noticed from the commencement was partly due, I believe, to cessation from violent movement whilst chloroform was being administered. Its final increase was possibly due to increasing pressure upon the brain. ${ }^{5}$

1 Niemeyer : Practical Medicine, vol. ii., p. 236, edit. 1880. Huguenin Ziemssen's Cyclopædia, vol. xii., p. 638

2 Tanner: Practice of Medicine, vol. i., p. 363, edit. 1875. Niemeyer : Practical Medicine, loc. cit.

New York Journal of Medicine, March, 1855.

4 Diseases of Nervous System. Rosenthal, 1879, rol. i, p. 16 Zremssen's Cyclopædia, vol xii, p. 650

5 Leyden : Virchow's Archiv., vol. Ivii., p. 519.

Sanitation at Wolverton.-On the 11th inst. the new sewage works for Wolverton were opened. They were commenced in April of the present year, and their estimated cost was $£ 4467$; but it is expected that they will be completed under that estimate. The purification of the sewage is attained by Lomax's quiescent precipitation system.

AT the meeting of the Wrexham Board of Guardians on the 18th inst. a hope was expressed that the advisability of the compulsory notification of infectious diseases in all parts of the Unitfd Kingdom would be fully discuse ed at the next Central Puor-law Conference, and a resolution passed.

\section{AGORAPHOBIA.}

\section{BY R. PROSSER WHITE, M.B., M.R.C.S}

IN the wide field of scientific medicine, research and demonstration are gradually hemming in and contracting the circle of functional disorders, and the onus of causation is placed upon some unsubstantial coccus or some undefined catalytic action. Functional disorders, however, do exist, and are perhaps increasing, as is proved by the frequent usage of words expressing derangements without inflam. mation, or constant structural change, such as neurosis, neurasthenia, hysteria, hypochondriasis, \&c., to which category this curious phase of nervous phenomenon, I think, belongs.

B. C- - aged about thirty, a professional gentleman, of active intellectual habits, whose previous health was good and whose family history shows no predisposition to nervous disorders, complains that for the last five or six years he has suffered from a nervous kind of light-headedness, gradually becoming more marked and troublesome. The attacks occur about twice a week, and are brought on by the ideas of space, vastness, height, depth, eternity, or such kindred subjects ; and these ideas, however conveyed to the brain, such as the viewing of a large building, or vast area, or in conversation, the description of a voyage, even thoughts of the infinite power of God, or a feeling of loneliness-all and each are able to produce upon him an acute attack which shows itself by pallor of face, a peculiar oppression at the back of the head, palpitation, large drops of perspiration upon the forehead, and a feeling of great dread and exhaustion, and sometimes, as he describes it, a feeling of impending madness. The general condition shows slight tendency to stoutness, with anæmia ; the lungs, heart, and kidneys are practically sound. He suffers very much occasionally from bilious attack, as be calls them, but otherwise is not troubled with headaches, tinnitus, deafness, or any stomach derangement. After an attack he feels sleepy and heavy, and sometimes passes a large quantity of urine; he says he feels nervous, and dreads the onset of another attack. $\mathrm{He}$ is occasionally troubled with spermatorrhœe, and reflexes of all kind are very sensitive.

These feelings and attacks may sometimes be avoided or lessened in severity by moral determination; deep concentration of thought will enable him to cross an open space or certain places which otherwise induce these attacks of dread; companionship relieves the feeling of loneliness and fear produced by the thought of taking a holiday in a part of the country new to him. The presence of a cart, even a stick or umbrella in the hand, persons, or trees, gives a sense of confidence when walking an unknown road. Cheerful and lively converaation, with a congenial companion, will always ward off the attacks.

This case is, I think, a typical example of that described in 1870 by Benedikt and in 1872 by Westphal, under the term "agoraphobia," and is, I venture to think, a not uncommon form of nervousness, though exceptional in its extreme forms; it is an essentially neurotic, as distin. guished from an hysterical, affection. ${ }^{1}$ It is rarely describrd in text-book". Quain's "Dictionary of Medicine" gives only a short article, written by Dr. Fred. Roberts, who does not think the complaint idiopatbic, but says "it is sequential to some other condition." What that other condition is he does not say. Dr. Maschede is mentioned as desciibing a case of clithrophobia ${ }^{2}$ in a young man who exbibited giddiness and oppression whenever he entered a small room or narrow space, and was obliged to camp out in the open fields and woods, and only in the depth of winter could he be prevailed upon to sleep in a large and airy apartment with all the windows open. Dr. Maschede also mentions two other similar cases. The author thinks that agoraphobia and clithrophobia ought to be classed under the same head, as in both cases the characteristic symptom is that the patient cannot by any means form an accurate conception of his surroundings.

Dr. Grainger Stewart, in a recent lecture upon "giddiness," thinks that these cases are closely allied to ordinary giddiness produced by what he calls and describes as "contradictariness of sensory impressions." "Normal

\footnotetext{
1 Gulstonian Lectures, by Clifford Allbutt, March 4th,
} Britieh Medical Journal, May, 1879. 